\title{
A rare solitary neurofibroma of the philtrum of upper lip
}

\author{
Gen Takahashi $^{1 *}$, Hiromichi Matsuyama ${ }^{2}$ and Kazuhiro Nakahashi \\ ${ }^{1}$ Takahashi Dental Clinic, 4-16-33-1F Bounoshima Minoh, Osaka 562-0013, Japan \\ ${ }^{2}$ Department of Oral and Maxillofacial Surgery, Matsusaka City Hospital 1550, Tonomachi, Matsusaka, Mie 515-8544, Japan
}

\begin{abstract}
Neurofibromas are relatively common benign tumors usually originating from peripheral nerves. Multiple neurofibromas are seen as part of von Recklinghausen`s neurofibromatosis. But, solitary neurofibroma is particularly a rarity in the Head and Neck. A 69-year-old woman was referred due to a complaint of swelling in the philtrum of upper lip with no other symptoms. Due to the suspicion of a tumor in the lip, MRI was taken and hemangioma was considered. A diagnosis of solitary neurofibroma was made through a surgical specimen by excisioned intra-orally by general anesthesia, from the lesion by histological examination and immunohistochemistry for the S-100 protein. And 4-year follow-up, she was free of symptoms and signs of recurrence were not detected.
\end{abstract}

\begin{abstract}
Abbreviations: MRI: Magnetic resonance imaging, H\&E: Hematoxylin-eosin, NF-1: Neurofibromatosis type 1.

\section{Introduction}

Neurofibroma is the most common type of peripheral nerve sheath tumor. It arises from a mixture of cell types, including Schwann cells and peri-neural fibroblasts. It may occur either as solitary lesions or multiple. As part of the generalized syndrome of neurofibromatosis which is usually neurofibromatosis type 1 (NF-1), also called von Recklinghausen's disease of the skin. The skin is the most frequent location for neurofibromas, but lesions of the oral are not common $[1,2]$.
\end{abstract}

The rate is estimated to be $25 \%$ of all neurofibromas in the head and neck regions and $6.5 \%$ in the oral cavity as solitary or multiple lesions associated with NF-1 [3]. Oral neurofibroma is affecting predominantly adults, showing no gender preference or prevalence in females [4]. Intraorally the various sites of occurrence in the tissue are tongue, intraosseous of jaw, buccal mucosa, palate and gingival have been reported $[3,5]$. Practically, the reported cases of solitary neurofibroma on the lip are few in the English literatures. Here we report a rare case of solitary neurofibroma of the upper lip.

\section{Case report}

A 69-year-old woman was referred to the Department of Oral and Maxillofacial Surgery at Matsusaka City Hospital because of a painless swelling on the philtrum of upper lip. According to the patient, the swelling had been present for 10 years. Her medical and family history was no insignificant.

At the initial extra-oral examination, circumscribing softy mass in the philtrum of upper lip was seen, and normal facial function was noted. Paresthesia in the upper lip was not observed. Intraorally this asymptomatic mass was covered by normal skin (Figure 1a) and normal mucosa (Figure 1b) of the upper lip. And mass was not fixed to the surrounding soft tissues on palpation.
Magnetic resonance imaging (MRI) displayed a well distinct circumscribed mass lesion in the region of the middle of upper lip without adhering to around the tissue in the lip (Figure 2a and Figure $2 \mathrm{~b}$ ). Due to the strong suspicion of a tumor in the upper lip, a surgical specimen was taken intraorally by general anesthesia (Figure 3 ). Formalin-fixed, paraffin-embedded surgical tissue was stained with routine hematoxylin-eosin (H\&E).

Microscopically, the tumor with well-defined margin, but not encapsulated, composed of interlacing bundles of spindle-shaped cells with wavy nuclei (Figure 4). These spindle cells were intermixed with delicate collagen bundles and variable amounts of myxoid matrix. Mast cells tend to be numerous. Capillary vessels can be seen among waved collagen fibers. Pathological diagnosis was a neurofibroma with no signs of malignancy.

In order to confirm the diagnosis, immunohistochemistry studies for S-100 on the lip specimens were performed (Figure 5) using the DAKO EnVision System according to manufacturer's instructions. It was found to be immunoreactive and the cells of tumor exhibited a scattered positive staining for antibody against S-100. Therefore, the mass was diagnosed as a solitary neurofibroma to the upper lip. After 4 year follow-up, she was free of symptoms and new mass were not detected.

\section{Discussion}

Oral neurofibroma is a benign tumor, which is the absence of symptoms and slow growth pattern ${ }^{6}$. In our case, the patient does not have a medical examination for 10 years until her friends aesthetically

*Correspondence to: Gen Takahashi, Takahashi Dental Clinic, 4-16-33-1F Bounoshima Minoh, Osaka 562-0013, Japan, Tel: +81-072-725-1182; Fax: +81072-725-1184; E-mail: gen1980@hotmail.com

Key words: solitary neurofibroma, lip, oral, neurogenic tumors

Received: May 28, 2020; Accepted: June 12, 2020; Published: June 15, 2020 

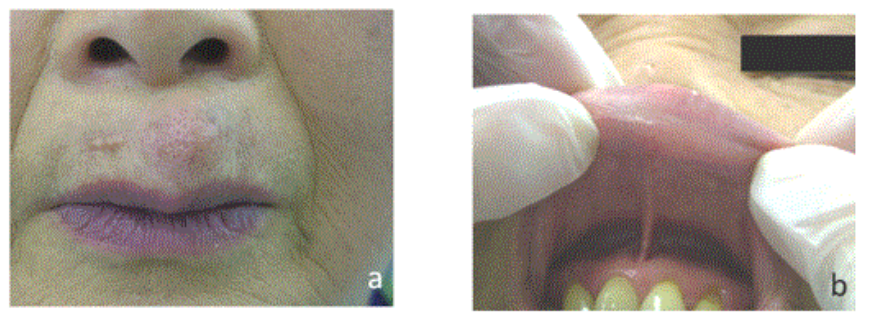

Figure 1. Circumscribing softy mass on the philtrum of upper lip. This asymptomatic mass was covered by normal skin (a) and normal mucosa (b)
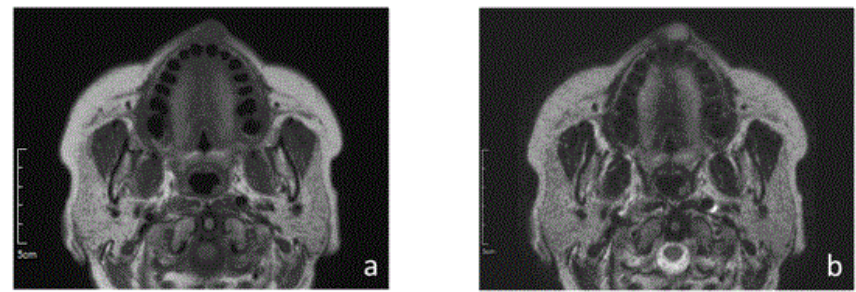

Figure 2. On axial MRI exhibits a mass in the region of the philtrum of upper lip. (a) On the T1, the lesion showed intermediate signal intensity: that is higher signal intensity than musculature, and lowers than fat tissue. (b) The T2 showed high signal intensity; that is, the similar signal intensity as well as that of fat tissue

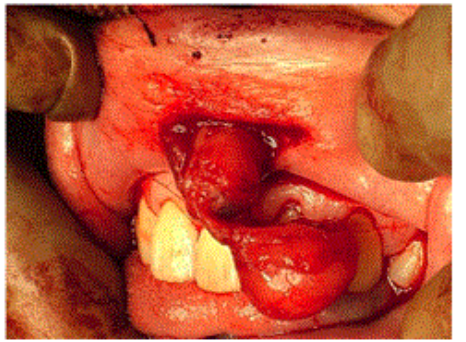

Figure 3. Surgically excised the tumor from the mucosa of upper lip

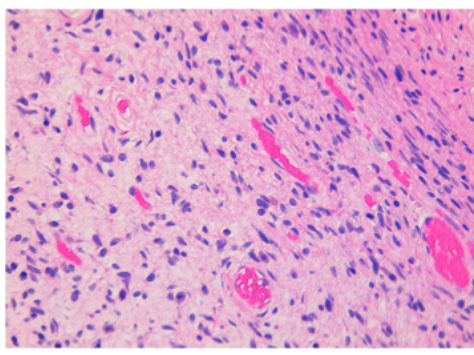

Figure 4. Photomicrograph of the excision of the lip shows neurofibroma composed of interlacing bundles of spindle-shaped cells with wavy nuclei (original magnification, $\times 40$; H\&E stain). These microscopic features are intermixed collagen bundles and myxoid matrix and capillary vessels in the stroma

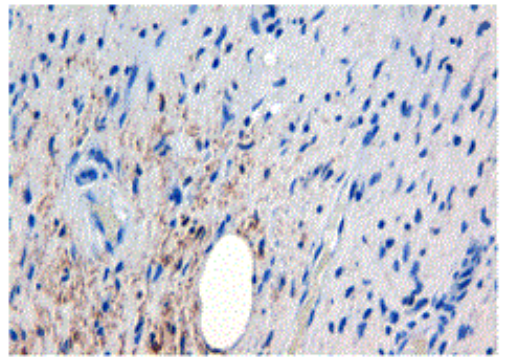

Figure 5. (a) Neurofibroma of the philtrum of lip (original magnification $\times 40$ immunohistochemical staining). The cells of this nurofibroma exhibit positive staining with antibody against S-100 pointed out the upper lip mass. So, the asymptomachic softy mass could be responsible for delay in the diagnosis, causing to a late search for treatment.

It is reported that most of the nurofibromas are associated with NF-1 and about $10 \%$ of patient with NF-1 develop malignant peripheral nerve-sheath tumors [7]. It is important diagnosis whether or not having NF-1, for the prognosis of the condition. NF-1 can be diagnosed through a family and medical history, as well as by physical examination [1]. Though malignant transformation of solitary neurofibroma is rare, the malignant transformation of a solitary neurofibroma in Head and Neck, which is not accompanied by NF-1 has been reported for as first a case report in 2010 [8].

Although the aetiology of solitary neurofibroma is still unknown, whether hyperplastic process or neoplastic, it is reported that all NF-1 patients harbor one nonfunctional NF-1 gene in every cell in the body. Then neurofibromas arise as a result of a second, somatic mutation [3]

In our case, the lesion of the upper lip was not encapsulated, with a low intensity layer on T1 and a high intensity layer on T2. Actually the fibrous capsule was not noted microscopically, which correspond to these MRI features. Differential diagnosis based on MRI findings includes hemangiomas, myxomatomatos tumor and schwannoma [9]. Due to the suspicion of a tumor in the lip, MRI was taken and hemangioma was initially considered differential diagnosis in our case. It is reported that differentiating hemangioma from neurofibromas solely may be difficult on the basis on MRI appearance [9].

Solitary neurofibroma is most frequently found in the tongue, gingiva, the alveolar mucosa, the plate, the buccal mucosa and the floor of the mouth [3]. However, lip cases are few in English literature (Table 1) [10-14]. Especially solitary neurofibroma of the philtrum of upper lip is not reported. Indeed, these 2 upper lip-cases are not located in philtrum, so in the labial mucosa of the upper lip. Although prevalence of lip site was not detected.

Upper lip location may lead to clinical misdiagnosis of solitary nurofibroma. It is reported that differential diagnosis of lip swelling, such as lipoma, angiolipoma, chondroid lipoma, myolipoma, hamartomatous lesion, schwannoma, lymphangioma, hemangioma, granular cell tumor, fibroma, leiomyoma, rhabdomyosarcoma, hemangioma, pleomorphic adenoma $[11,14]$.

However other literatures report that lip is common site for solitary neurofibroma [4]. These reported lip cases are grounded early literatures, which is not so neurofibroma as solitary neuroliemmomas. Because of confusion in the early literature as to the cell of origin, compounded by the interchangeable usage of the terms neurilemmoma and neurofibroma, has perpetuated a great deal of uncertainty about these lesions until 1978 [5].

Histopathologic features of solitary and multiple neurofibromas are essentially identical [5], although one literature reported that the solitary neurofibroma is well demarcated from the surrounding connective tissue in contrast to many NF-1 [15]. The neurofibroma cases are positive stain for S-100. However, the S-100 immunostain in neurofibroma is not as notable as in neurilemmoma [16]. The main differential diagnosis of spindle cell tumors on the face and the oral is included nodular fasciitis, low-grade myofibroblastic sarcoma and dermatofibroma. In our case contains spindle cells with wavy -shaped nuclei distributed on a background of loose connective tissue matrix which is myxomatous and the tumor with well-defined margin, but not encapsulated. The tumour cells are uniformly positive for S-100 protein. Therefore, the lip tumor was diagnosed as a neurofibroma. 
Table 1. Clinical finding of 6 cases of solitary neurofibroma of lip

\begin{tabular}{|c|c|c|c|c|c|c|c|c|}
\hline Case No & Age & Gender & Lip Site & Clinical Diagnosis & Size & Treatment & Follow Up & Author \\
\hline 1 & 19 & F & Upper right labial mucosa & sebaceous cyst & Unknown & surgical excision & Unknown & J. Traiger (1966) \\
\hline 2 & 14 & F & Upper right labial mucosa & Unknown & $10 \times 6 \mathrm{~mm}$ & surgical excision & 1 year & P. López-Jornet (2010) \\
\hline 3 & Unknown & Unknown & Lower lip & Unknown & $10 \times 30 \mathrm{~mm}$ & Unknown & Unknown & Y. B. Lee (2012) \\
\hline 4 & 12 & $\mathrm{M}$ & Lower middle labial mucosa & Unknown & $6 \times 3 \mathrm{~mm}$ & surgical excision & 1 year & A. H. Borges (2013) \\
\hline 5 & 43 & F & Lower right labial mucosa & Unknown & $7 \times 5 \mathrm{~mm}$ & surgical excision & 2 years & M. Kouhsoltani (2015) \\
\hline 6 & 69 & F & Philtrum of upper lip & hemangioma & $15 \times 15 \mathrm{~mm}$ & surgical excision & 4 years & Our case \\
\hline
\end{tabular}

Most of solitary neurofibroma is good prognosis and rare local recurrence after excision. However neurofibromas have been reported to locally recur after treatment more often than schwannomas. The absence of a capsule in neurofibroma is making complete extirpation of the tumor more difficult [17]. In our case, After 4 year follow-up, she was free of symptoms and new mass were not detected. Also, our case had no other evidence of multiple lesions. It is possible that this solitary neurofibroma of lip may be the first manifestation of NF-1 in the future. A solitary neurofibroma of the lip are rare and are reported knowing the long-follow-up cases, so regular clinical long-follow-up is needed.

\section{References}

1. Apostolidis C, Anterriotis D, Rapidis AD, Angelopoulos AP (2001) Solitary intraosseous neurofibroma of the inferior alveolar nerve: report of a case. Journal of Oral and Maxillofacial Surgery 59(2): 232-235.

2. Toth BB, Long WH, Pleasants JE (1975) Central pacinian neurofibroma of the maxilla. Oral Surgery, Oral Medicine, Oral Pathology 39(4): 630-634. [Crossref]

3. Marocchio LS, Oliveira DT, Pereira MC, Soares CT, Fleury RN (2007) Sporadic and multiple neurofibromas in the head and neck region: a retrospective study of 33 years. Clinical Oral Investigations 11(2): 165-169. [Crossref]

4. Campos MS, Fontes A, Marocchio LS, Nunes FD, de Sousa SCOM (2012) Clinicopathologic and immunohistochemical features of oral neurofibroma. Acta odontologica scandinavica 70(6): 577-582.

5. Richards D (1983) Neurofibroma of the oral cavity. British Journal of Oral Surgery 21(1): 36-43.

6. Neville BW, Damm DD, Allen C, Bouquot J (2008) Oral and Maxillofacial Pathology.
7. Mautner V, Friedrich R, Von Deimling A, Hagel C, Korf B, et al. (2003) Malignant peripheral nerve sheath tumours in neurofibromatosis type 1: MRI supports the diagnosis of malignant plexiform neurofibroma. Neuroradiology 45(9): 618-625.

8. Chen H-J, Chen H-S, Chang Y-L, Wu Y-Y (2010) Malignant peripheral nerve sheath tumor of the neck: transformation from a recurrent neurofibroma in a patient without neurofibromatosis. Tzu Chi Medical Journal 22(4): 195-199.

9. Sakata A, Hirokawa Y, Kuwahara R, Hamada A, Kuroda M, et al. (2013) Solitary oropharyngeal neurofibroma: MR appearance with pathologic correlation and review of the literature. Clinical Imaging 37(3): 554-557. [Crossref]

10. Traiger J (1966) Solitary neurofibroma of the lip: Report of a case. Oral Surgery, Oral Medicine, Oral Pathology 21(2): 148-150. [Crossref]

11. López-Jornet P, Gomez-Garcia E, Camacho-Alonso F (2009) Solitary oral neurofibroma. The New York State Dental Journal. 76(5): 54-55.

12. Lee YB, Park HJ (2012) Letters to the editor: Solitary neurofibromas: Does an uncommon site exist? Annals of Dermatology 24(1): 101-102.

13. Borges AH, Correia RDM, Borba AM, Guedes OA, Estrela CRDA, et al. (2013) Unusual solitary neurofibroma on the lower lip of a child. Contemporary Clinical Dentistry 4(4): 512. [Crossref]

14. Kouhsoltani M, Taghavi Zonooz A (2015) Solitary Neurofibroma of the Lip: Report of a rare case. Res Dent Sci 11(4): 247-250.

15. Wright B, Jackson D (1980) Neural tumors of the oral cavity: a review of the spectrum of benign and malignant oral tumors of the oral cavity and jaws. Oral Surgery, Oral Medicine, Oral Pathology 49(6): 509-522. [Crossref]

16. Chrysomali E, Papanicolaou SI, Dekker NP, Regezi JA (1997) Benign neural tumors of the oral cavity: a comparative immunohistochemical study. Oral Surgery, Oral Medicine, Oral Pathology, Oral Radiology, and Endodontology 84(4): 381-390. [Crossref]

17. Polak M, Polak G, Brocheriou C, Vigneul J (1989) Solitary neurofibroma of the mandible: case report and review of the literature. Journal of Oral and Maxillofacial Surgery 47(1): 65-68.

Copyright: (C2020 Takahashi G. This is an open-access article distributed under the terms of the Creative Commons Attribution License, which permits unrestricted use, distribution, and reproduction in any medium, provided the original author and source are credited. 